Review scientific paper

\title{
ROLE OF OPERATOR TRAINING SIMULATORS IN HYDROCARBON INDUSTRY - A REVIEW
}

\author{
Kallakuri, R. \& Bahuguna, P. C. \\ School of Business, University of Petroleum and Energy Studies, Dehradun, Uttarakhand, India \\ E-Mail: foralldata@ hotmail.com, bahuguna@ddn.upes.ac.in
}

\begin{abstract}
Control room operators in the hydrocarbon industry monitor large scale process data and take corrective actions using multifaceted control systems. Simulator training provides them an understanding of how to accurately identify process upsets and quickly respond to process control issues before they escalate to shut down of units. Although operator training simulators are widely used in the industry, they are not regularly updated to latest features and become obsolete due to different stakeholder issues. The objective of this review is to examine these attributes in the literature to identify key perceptions about simulator training and suggest appropriate resolutions. The findings accentuate the need to develop training simulators integrated with the actual process configuration, accurate process modelling, precise feedback mechanism, training data analysis in conjunction with operations and continuous learning assessment showing embedded training value to the organization. This approach of using instructional design and pedagogical methodology can contributes for effective usage of simulators and might help to minimize human errors in the hydrocarbon industry.

(Received in August 2021, accepted in October 2021. This paper was with the authors 1 week for 1 revision.)
\end{abstract}

Key Words: $\quad$ Operator Training Simulator, Simulator Configuration, Human Error, Training Transfer

\section{INTRODUCTION}

Process operators monitor, control and operate process and plant equipment by different unit operations using Integrated Safety and Control Systems (ICSS). They are normally classified as field operator, the person working inside the process area or field and control room operator, the person working inside the control room. Operator jobs are considered unique and their training is historically perceived to be beyond a cost-effective outsourced training approach. The training of operator mostly takes place, while they are on the job, known as OJT (On the Job Training). The ever-increasing and continually improving automation in the industry leads to sophisticated processes that are more complex for the operators to understand. Consequently, the sophisticated processes tend to reduce human reliability. The implementation of new control systems and enhanced automation leads to complexity of control room operations. This brings to fore possible acclimatization issues faced by workers who may have a high level of familiarity with legacy systems from their experience and habitual in handling the operations [1]. There are several challenges unique to the development of technical workers as the workplace knowledge is outdating quickly and workers face skill obsolescence unless their knowledge is updated in a pedagogical process. This requires competence in how to learn and a work climate that encourages real-time learning to solve problems and the supportive work climate depends on what employers and managers do to encourage expansion of knowledge [2]. New generation operators are more comfortable working with advanced automation technologies and so helping organizations to take advantage of the productivity and performance benefits available.

Operator Training Simulator's (OTS) are used in the hydrocarbon industry to train the operators for both routine operations, start up, shutdown and abnormal situations related to the process. The benefits are repeated training on rare events, process upsets, validate or test new procedures, practice start up etc., which in turn increases confidence, enhances their effectiveness and decreases work-related stress in executing the tasks. Although OTS exists in 
many industries, there is lack of proper usage due to various issues pertaining to availability of competent instructors, operator's willingness and or reluctance to participate and their interest in OTS training, effectiveness of training sessions, updating the OTS in tandem with actual process, management support and budget. These will play an important role to keep them in proper working conditions to get desired results and strive for success. The abnormal situations, in particular, during the start-up, unscheduled shut-down are subject to a very substantial part of the significant incidents occurring in many plants. The recurring accidents in the process industry are due to lack of learning from earlier incidents, awareness and applying the lessons learnt from the accidental reports into the training of operators. Human errors and errors related to human factors related to training, its effectiveness and challenges faced by different stake holders continue to contribute to losses in the industry.

The objective of this study is to examine these attributes with literature from different sectors and industries using training simulators for training to: Identify role of key stake holders? Ways to prevent OTS obsolescence? How the best practices from other industries can be used in hydrocarbon industry? How these efforts can be further enhanced by management intervention? The aim is to improve operator competency, minimize human errors and maximize productivity of OTS training. This review examines the key relationships between different stake holders of the OTS, like process operators, instructor, supervisors and simulation engineers etc.

\section{LITERATURE REVIEW AND METHODOLOGY}

The purpose of the study is to analyse the existing literature on the operator training simulators, justify the investment in terms of their training efficacy, their usage in the industry as a training tool, perception of different stake holders, how to prevent their obsolescence and increase their usage. The literature is reviewed using the eight step Systematic Literature Review (SLR) process. SLR is defined as a systematic, explicit and reproducible method for identifying, evaluating, and synthesizing the existing body of completed and recorded work produced by researchers, scholars, and practitioners [3]. First the different key phrases and words recurring from different oil and gas literature, training industry and commonly available databases are collected.

The primary literature review findings are analysed, papers are filtered to refine the keywords to analyse data and further they are combined under different themes to enhance the scope for further research. The different themes derived from the primary literature are filtered by an online focus group discussion consisting of ten industry professionals, consisting of four training specialists /instructors, two plant managers and three senior control room operators along with the author in over two meetings of two hours each. Structured interview with checklist and questionnaire is used to modulate the session [4]. Finally, six different themes for further review of literature along with their search outlook are validated by existing literature and are presented in Table I and role of operator training simulators with reference to these themes is summarized in the next section.

\subsection{Role of operator training simulators}

Simulators are used in chemical operations and process trainings, industrial safety components design, process modelling and testing. They are also used in areas such as commercial and military aviation, shipping, military, and first responder agencies etc. They provide a safe substitute in replicating the very high-risk and often complicated scenarios. Real world renditions of these situations are too costly and dangerous to provide opportunities for staff to repeatedly practice and be competent in the highly stressful workplace situations. 
Table I: Literature review with themes and search outlook.

\begin{tabular}{|c|c|}
\hline Themes & Search outlook in the literature \\
\hline $\begin{array}{l}\text { Human errors in process } \\
\text { control [5] }\end{array}$ & $\begin{array}{ll}\checkmark & \text { Human errors in process control } \\
\checkmark & \text { Situational awareness during process control } \\
\checkmark & \text { Conditions leading to the events/accidents } \\
\checkmark & \text { Process incidents involving human errors } \\
\end{array}$ \\
\hline Process operator training [6] & $\begin{array}{l}\checkmark \text { Operational errors during the process control } \\
\checkmark \text { Operator competency } \\
\checkmark \text { Training of operators in the oil \& gas industry }\end{array}$ \\
\hline $\begin{array}{l}\text { Operator training simulator } \\
\text { training [7] }\end{array}$ & $\begin{array}{ll}\checkmark & \text { OTS usage, limitations and modelling } \\
\checkmark & \text { Underutilization of OTS } \\
\checkmark & \text { Instructor role and responsibility } \\
\checkmark & \text { Simulation complexity } \\
\checkmark & \text { Process modelling } \\
\end{array}$ \\
\hline Training effectiveness [8] & $\begin{array}{ll}\checkmark \text { Effectiveness of training } \\
\checkmark \text { Training evaluation methods } \\
\checkmark \text { Training feed back } \\
\checkmark \text { Competency \& skills improvement } \\
\end{array}$ \\
\hline $\begin{array}{l}\text { Training transfer \& learning } \\
\text { transfer [9] }\end{array}$ & $\begin{array}{ll}\checkmark & \text { Training transfer through simulation } \\
\checkmark & \text { Training \& learning transfer methods } \\
\checkmark & \text { Applying knowledge in workplace } \\
\checkmark & \text { Enhancement of the current practices }\end{array}$ \\
\hline
\end{tabular}

The skills of the operators can be sustained with proper training of the simulated scenarios and practice them repeatedly using a suitable training simulator [10]. The overall goal of training via simulation is to build confidence in staff in their ability to perform under adverse conditions [11]. Further the behaviour of the operator is influenced by many external factors like work surroundings, personal factors and environment, which necessitates human factors should be considered while designing a simulated process [12]. Simulator training can be further enhanced with the application of suitable learning stratagems. One can select them based on the characteristics of the training group and theme or simulation process to be trained or configured in the training simulator. Training is defined as the planned learning experience that trains the employees how to perform the current and future jobs. Workplace learning improves the profitability of different departments while cultivating more positive attitudes toward profit orientation across an organization. Mostly the training is focused on what needs to be known, rather than the application of skills.

The objectives of training determine the most appropriate criteria for assessing the effectiveness of training [13]. The main goal of training is to prepare participants for the tasks they are going to perform on their jobs [14]. Learning is the process of acquiring the ability to respond adequately to a situation which may or may not have been previously encountered. This means it is a change that occurs in our behaviour, more specifically in our work behaviour because of some experience or practice. The training must focus on this change in behaviour, which gets reflected in performance and variable-priority training led to better multitasking performance, and a trend for a reduction in the automation complacency effect [15]. One must be motivated and willing to engage completely in the learning process to recognize their weaknesses in decision-making and clinical reasoning skills and to be humble enough to correct those weaknesses with the guidance of the instructor [16]. There is a need for developing an improved methodology to train process operators efficiently as their performance has a direct influence on the safety, productivity, profitability, stability, and controllability of the process [17]. With many similarities with the military, medical, transport and aviation industries, the 
simulation training effectiveness research in these sectors can be applied in the oil \& gas industry. Due to the similarity of training delivered in various businesses, like Aviation, Automobiles, Business Chemical industry, Education, Gaming, Medical, Military, Mining, Marine, Shipping, Nuclear, Oil \& Gas, Petrochemicals and Power sectors etc. The usage of training simulators, their training effectiveness, reduction of human errors due to training interventions are being looked upon in the research.

The simulation literature lacks significant research how the different elements of simulation training can be used to create a learning environment that encourages active learning and engagement in the workplace. The training evaluation and effectiveness on the individuals or across operations teams is not studied in the perspective of an operator across the industry using available models.

\subsection{Methodology}

Exploratory research methodology is used as the main basis of this review. Exploratory research with literature review can add quality and insightful information to a study and allows the researcher to be inventive and to gain insightful visualization of a particular subject. The review of literature is carried out using Preferred Reporting Items for Systematic Reviews and MetaAnalyses (PRISMA) methodology. PRISMA is an evidence-based minimum set of items for reporting in systematic reviews and meta-analyses. This is primarily focused on reporting of literature reviews and evaluating the effects of interventions. This is used to gain an understanding of primary reasons, thoughts, and inspirations among the different stake holders. This can also be used as a basis for reporting systematic reviews of literature for further research and with clearly defined objectives other than assessing interventions [18]. This brings structure to the review rather than coupled with the automated reviews of data bases. The study was made to include the most articles possible, but the present research also not to claim that the database is also not complete. Based on the literature review of around 80 papers using systematic review protocol and the percentage of articles related to themes from the articles are in Fig. 1 and subsequent segregation and analysis, shown in Fig. 2.

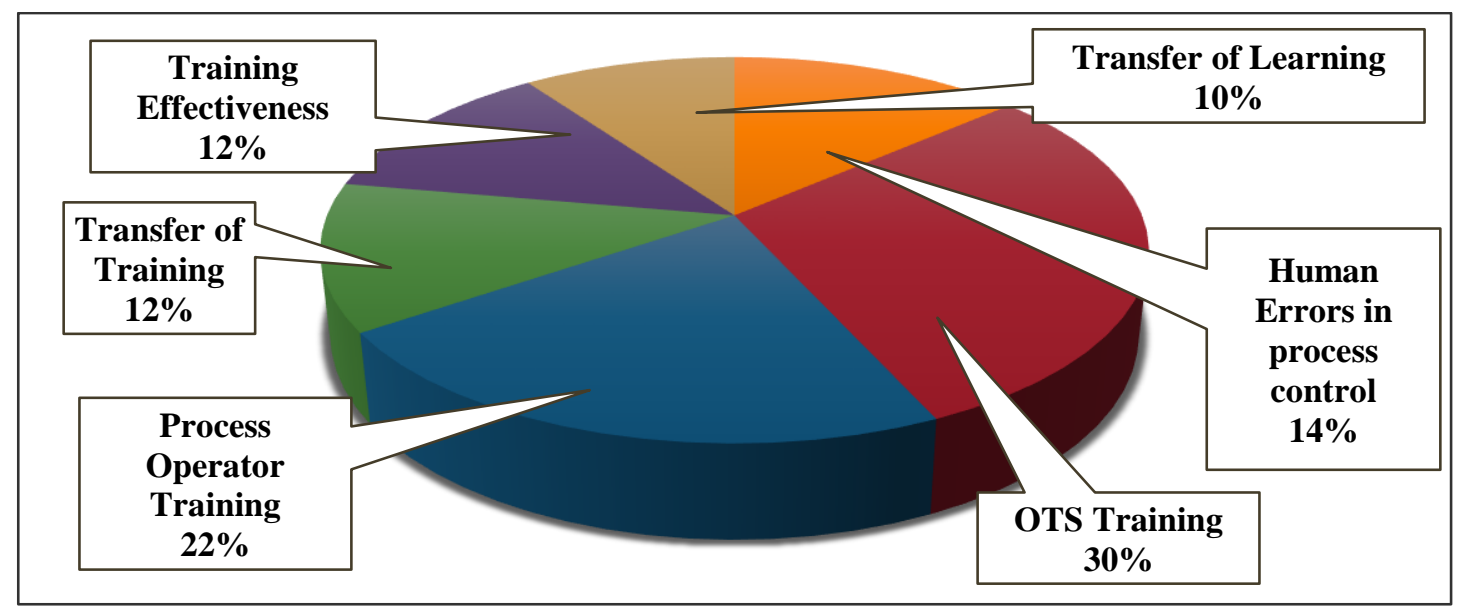

Figure 1: Themes vs Percentage of articles from the literature review.

The articles during the literature review are analysed using NVivo (trail version 12), Qualitative Data Analysis Software (QDAS) tool from QSR international. NVivo tools provide visual connections, patterns and themes so that researchers can visualize and analyse the connections and write in more apposite manner [19]. The word cloud in Fig. 3 shows that the words appearing in more size, bold and highlighted, are directly correlated to usage frequency and become small and shaded are of lowest frequency in the papers. 


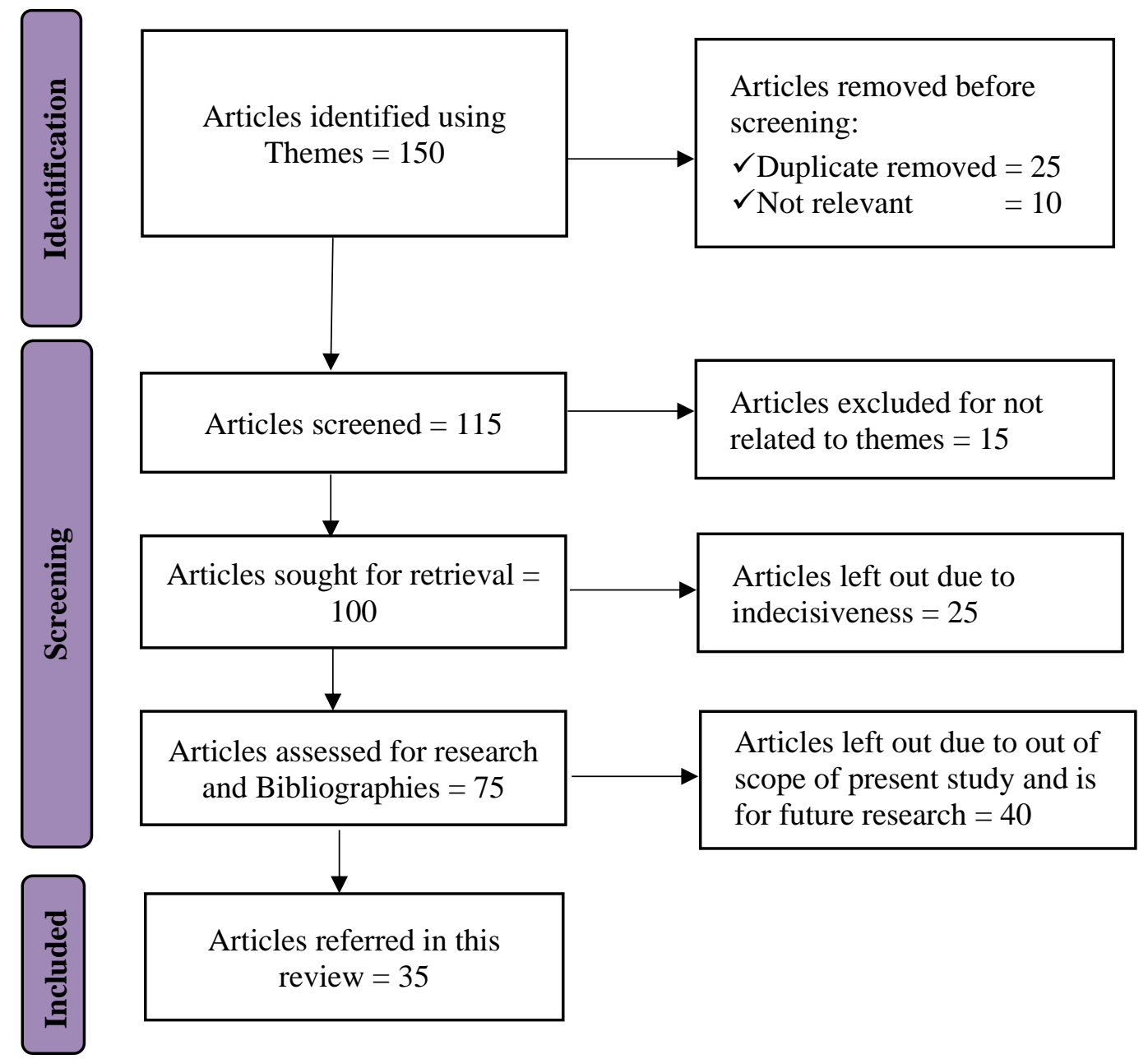

Figure 2: Literature review of research papers via online databases.

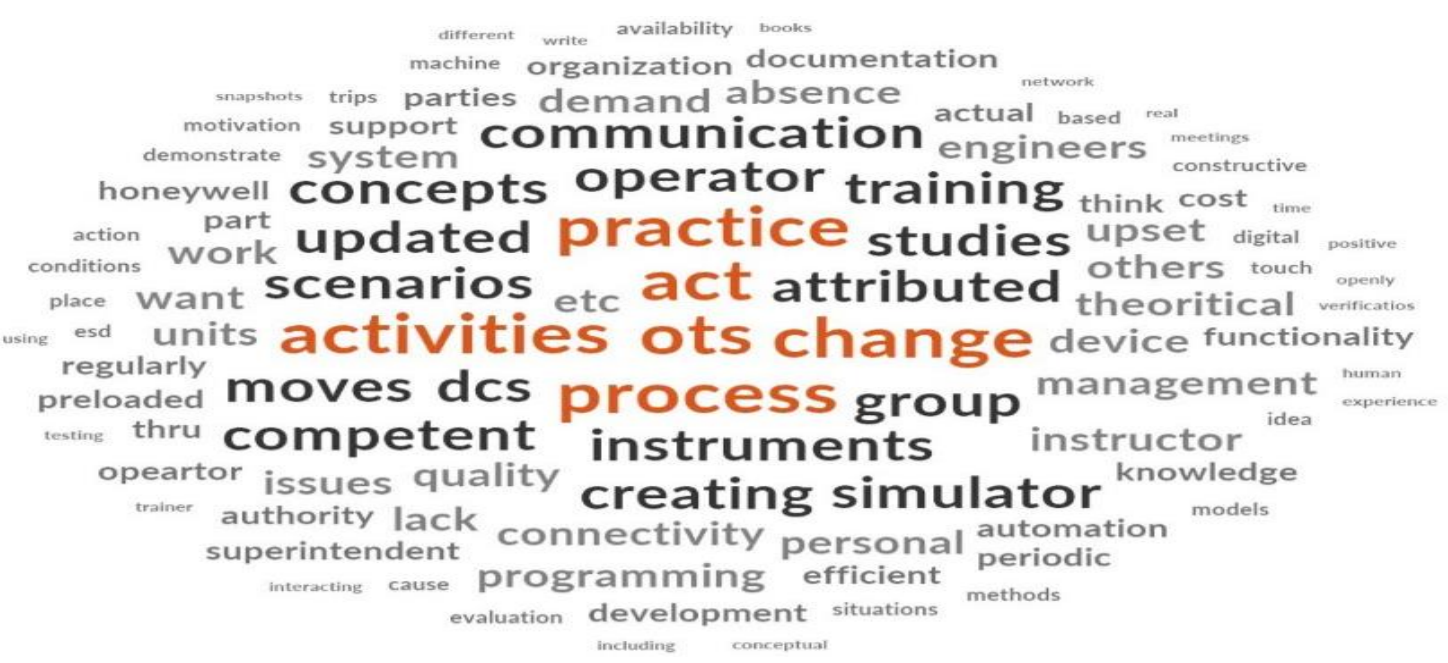

Figure 3: World cloud showing keywords and themes to visualize literature.

The tree map output in Fig. 4 visualizes that the larger rectangle is with highest coded words tabulated with the text representing themes and subtext (subthemes) representing relationship with the themes. These will help us to provide guidance in interpretation and demonstrate the coded themes and subthemes to validate findings, produce discussions with evidence. This also help in data captured in phrases and words for revisiting for future updates and revisions. 


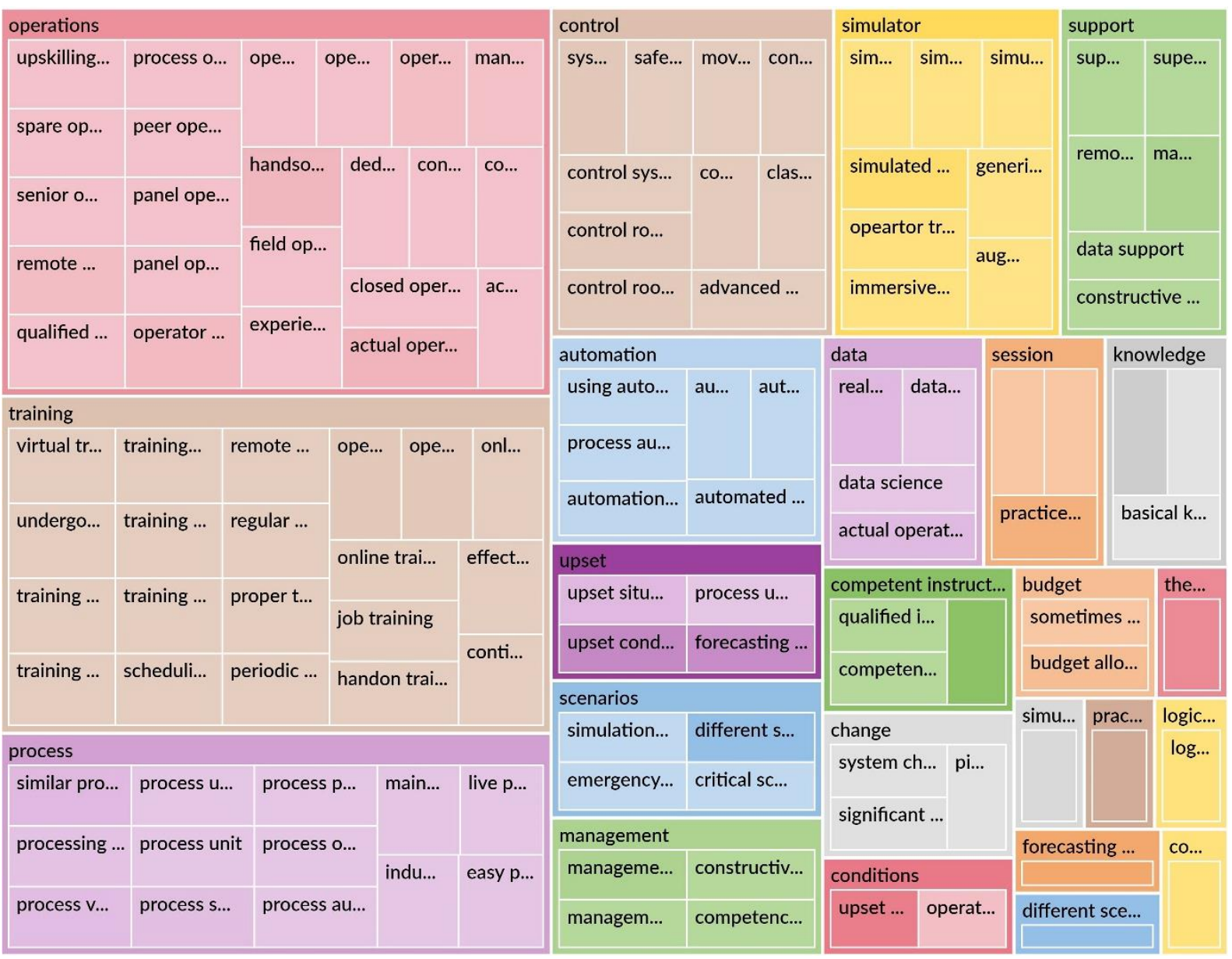

Figure 4: Tree map with themes and key words.

The next section summarizes main points of the thematic literature review and analysis is in three broad subsections, namely: i) Effectiveness of OTS training, ii) OTS training phases and its usage and iii) Role of key personnel. This is done to ensure that the relevant topics are discussed and grouped in alignment with the objectives.

\section{DISCUSSION}

The training literature review shows that the four key features of effective simulator training are accurate process modelling, precise feedback mechanism, training data analysis in conjunction with operational errors (from the actual operations) and continuous learning assessment of operators by the instructor. The sub themes, namely data from operational errors, basic technical knowledge, logical relationships between different scenarios, forecasting unit upsets and practice, technical knowledge revision along with improvements in process modelling further validate the fact that the simulator can be used to identify the most frequent human errors or operational errors taking place while implementing a procedure or testing a control loop etc. This agrees with earlier findings that the procedures can be optimized to insist on critical points, reinforce learning by repeated practice and then the chances of errors are less, as the operators already practiced and be confident in executing the complex tasks [20]. The confidence in simulators might be due to the fact that most research on their effectiveness has been based on the subjective evaluation of trainees rather than on objective performance data [21]. There is a need to develop robust training practices, which are adaptable to an ever changing and technology incentive working environment [22]. The reduced incidences of 
accidents and resultant downtime, in the case of both personnel and equipment, means an increase in productivity, which justifies the investment on the simulators.

\subsection{Effectiveness of OTS training}

To know that the training program or session effective, it needs to be well-defined in a clear evaluation criterion and gauging its impact on specific individuals or organization. It is to be tested and feedback is collected by using pre-defined methodology, either by manually or automatic means, without any bias towards the topic, instructor and other organizational issues. The key points for maximum utilization of OTS and there by its effectiveness are a) Classroom training followed by training $(25 \%)$, b) Process models with up to date simulator $(22 \%)$, c) Well planned training sessions with qualified instructors (19\%) and d) Support from management $(15 \%)$ in a OTS training effectiveness survey [23]. The instructors, by virtue of their operational background are not formally trained on evaluation techniques and assessment tools. The effectiveness of a training program depends on how effectively the skills are transferred to the trainee [24]. Effective learning transfer is vital to ensure that the planned learning outcomes from training are captured during the session and transferred to workplace. This is often questioned as most of what is learnt from training programs is not transferred to the workplace.

Training evaluation is considered as an ongoing process, rather than done only at certain intervals. A number of barriers exist that prevent organizations from evaluating operators at various levels. The three major reasons to evaluate training programs are i) to improve the program, ii) to maximize transfer of learning and achieving subsequent organizational goals and iii) to demonstrate the value of training to the organization, which is effectiveness. In addition to tangible benefits, most training programs will have intangible benefits and the key challenge is to effectively identify them. There is significant change in the attitude, skill level and knowledge due to the usage of simulation training of sea farers and Kirkpatrick's model found to be most suitable model for measuring the training effectiveness of different training courses and having different sessions [25]. The ARC survey, OTS best practices revealed that more than $80 \%$ of the participants, consisting of top and middle management, instructors and operators across the world, believes that simulation-based learning is viewed as a strategic component of the overall training policy of the company [26].

\subsection{OTS training phases and its usage}

Although OTS are widely used across the industry for operator training, mostly the benefits are seen in the commissioning phase of training. This is due to fact the operators are new to the process and OTS is the only visual and hands on tool to view operating conditions along with the graphics, which represent the actual process flow conditions. The immediate benefits to the management and supervisors include less time to start up, optimizing operating conditions to reach product quality and quantities as per the design. OTS is also widely used to check control loop configuration and tuning during this phase. Once operators are accustomed to the process conditions and able to achieve start-up of the units in a more proactive way as they already know the different conditions in the OTS and actual plant. Sometimes modifications carried out in the actual process on the basis of operating conditions cannot be captured. The operators are also reluctant to go back to the OTS and practice, as they do not have more to learn and easily demotivated to use their time for OTS refresher training.

There are three primary concerns in the initial development of training simulator. They are, Fidelity (degree to which a simulation replicates real life), Interactivity (level of user interaction with the simulation) and Immersion (extent to which a user is cognitively, physically, and affectively engrossed in the simulation) [27]. As the level of fidelity, interactivity, and 
immersion in the simulator configuration increases, the cost of developing the simulator increases. The management has to spend resources in the initial phases without thinking of immediate return of investment as the benefits are long-term and not directly measurable in dollar terms. The opportunity to study the modelled virtual process, structure and content of the library of logics and algorithmic diagrams of the typical control functions is useful in a simulating environment to test (tests such as loop tuning, acceptance of advanced control configurations etc.) and validate before being used in the actual distributed control systems during start up and revamp of existing facilities [28]. On the other hand, the cost of simulator development must be weighed against the cost of acquiring actual systems for practice and/or the cost of physical experimentation on actual equipment or in a real-life situation. One can also calculate the recurrent cost of sending required number of operators to another site or similar processes for training and initial investment needed for a training simulator with the number of operators to be trained. One of the major discernments among the managers is that a simulator is a luxury that cannot be justified in financially hard times. A simulator does not train automatically, it has to be used by manual intervention of the instructor to extract the benefit, yields the benefit and the desired results for the management.

One of the most productive way of configuring the simulator is to incorporate intelligence in the initial design and rules-based functionality using technical information available at that time [29]. What is more important than the simulator technology is that how educational methodologies to be applied and whether it increases training effectiveness significantly or not. This has some issues as often the simulator trainer is not properly trained to use pedagogical tools and updated learning methodologies as he is often selected internally without any external recruitment and most of the time he is senior most among the many control room operators present in the organization. Even though some companies have the most modern and upscale simulator with full scale simulation of the units along with safety shutdown systems, using it for training and maintaining them over a period of time is a continuous effort of both the instructor, operations and automation teams. The operations team, being the end user also have to follow up for OTS maintenance, tracking its usage and provide necessary resources for OTS maintenance and upkeeping.

\subsection{Role of key personnel}

The OTS instructors perform a variety of roles like planning, tutoring, managing trainings along with sustaining the system ready for training all the time. They further facilitate training materials, carryout lectures and role-plays the scenarios. The instructor can create an interactive environment, where in instructor and students actively participate in a demonstration, using the OTS applying theory to the real-world scenarios. The Instructor is responsible for designing and managing an effective simulation training session. During simulation, the instructor creates multiple learning experiences such as pre-briefing, session preparation, the actual simulation scenario and debriefing. Instructors bring knowledge, expertise, expert clinical reasoning skills, and maturity to the environment. In the debriefing, it has to be stressed that the real time situations have their own complexity and professional intervention is needed to control the process/operations so that the process remains operational without any untoward incident or loss of production [30].

The OTS Instructor can change the process parameters (variables) and simulate conditions for unit operations as required to train the operators. The instructor must possess both content knowledge and instructional skills for teaching in a simulated environment. Standards or guidelines defining instructor qualifications are necessary to ensure instructional effectiveness. The availability of fully competent instructors is limited, which also limits the number of operators that can be trained at the same time. They are expected to maintain constant communication with plant operations personnel in shift operations, so that they can maintain 
operational expertise and familiarization with plant procedures [31]. The simulators also allow trainees to make actions and review the impact of their actions with the instructor. This is a critical part of learning that is not possible using OJT activities and thereby OTS provides a clear advantage over the other type of operator trainings. For the operators, the benefits come from repeated training on rare events and process upsets, validating new procedures, which in turn increases confidence, enhances effectiveness and decreases work-related stress. One approach employs triangulation, use of multiple data sources and methods to gather data from prospective end users and combine qualitative data (e.g., from focus groups, interviews, and observations) with various forms of quantitative data (e.g., those from controlled study situations, process type, with or without safety systems etc.) [32]. The root causes of many industrial accidents and their corrective action reports in the industrial literature are linked to the human performance and suitable training to improve the competency and performance.

Site managers are recommended to ensure that instructor training replicates the nontechnical and technical skills along with other instructional competencies required to ensure that the instructors are able to fully support growing facility requirements and be able to establish credibility with trainees on a continuous basis [33]. The operations, automation, process and engineering sections have to facilitate the maintenance of OTS in tandem with instructor. The management have to make sure this approach is similar to the actual plant control system if both are supplied by same vendor, if not they need to work along with both suppliers in arranging continuous support either offline or online. The supporting staff should be accessible to the instructor and needs to be trained first before site installation. This way we can close the gaps between the actual systems and simulator models, even carryout modifications first in the OTS, test and validate the results before being implemented in the actual process.

The training simulator does not guarantee a successful training unless a program based on a systematic approach to training is used. Opinion-based surveys, questionnaires and checklists are best used in combination with objective measurements (e.g., time, speed, error) of trainee performance. The positive results from these methods may not guarantee that the simulator training is effective unless one is addressing the needs of the operators in a more tangible way such as reduction of human errors, procedural modifications proposed and implemented after OTS training, validating start up time reduction from earlier start up and data collection for future comparative analysis and validation. These are elaborated in terms of operational modifications, implications to the industry, contribution to the society in the next section.

\section{CONCLUSIONS AND FURTHER RESEARCH}

A combination of skilled instructors, immersive simulation, cloud technologies coupled with up-to-date high-fidelity simulators and pedagogical course material are essential for ensuring the best possible training for the new and experienced operators in the hydrocarbon industry. Having an integral training centre with adequate personnel and material infrastructure is helpful to exploit both, simulators and training system [34]. The first step to show the value of a training program is to create a program that has organizational value embedded with results. The trainee's capabilities, personality, motivational factors, previous work experience will affect the learning process. Training of non-technical skills may help reduce the risk of human error and trained personnel are more prepared to react in case of an emergency situation [35]. Maintaining the work force competences is a challenge for the organizations and simulation training continues to be the best among the available trainings for operators. The operators are confident in executing the complex tasks, leading to product quality, skill improvement and one might see a reduction in human errors due to the competency-based approach. The operators have to take objective based decisions based on real time data, strive to contribute process 
changes, and needs to be competent for the ever-changing new cyber security and automation protocols.

The instructor role is changing due to the advent of new virtual and augmented technologies, highly automated with objective based decisions tools. They also need to be trained to update with the technology, which is often missing due to various organizational reasons. It was also found that the issue of whether the OTS is asset to the organization or lying like an underutilized asset mainly depend on the Instructor. In many cases instructor's overall technical evaluation is considered for modifying plant operating procedures and many instructors are able to assist with plant operation when needed in case of emergencies as often the simulators are based in the control rooms.

Instructors may also assist in incident investigations and knowledge management, or the preservation of knowledge that might be lost as a result of the retirement of very experienced and competent personnel, this way they have good rapport with different operations team and know first-hand information of each operator(s), their strength and weakness. The role of the instructor in evaluating, executing, integrating and supporting these needs to be clearly understood, and the appropriate support to be provided by the management. It is also important to recognize that available technologies change quickly, and arrangements and the capability need to be in place to ensure that these are suitably assessed, and a robust management of change process also need compliment the OTS changes.

Training effectiveness will depend on a number of factors, such as time spent, motivation, learning efficacy and cognitive ability. Behavioural based standard assessment of operators is more important to mitigate the risk of human error. This needs to be integrated with the modern simulators as a standard option, which will reduce the dependency on the instructor and same can be applied to all training sessions using the same simulator. The training session effectiveness may also reduce the cost to the company when the participants are able to understand the process effectively and maintain their competence at workplace for fast/efficient start up and limited downtime in case of a process upset or shutdown. By integrating the training evaluation models with the OTS, customized for each process during site installation, the instructor can easily measure each and every session without being dependent on questionnaires and manually inferring the results. This methodology can optimize the trainings delivered and improve future sessions.

These approaches will make them ready for next generation operations consisting of virtual, augmented, distant and unmanned operations where automation takes the centre stage with minimum human interference. Further research in the simulator training intervention methodologies and efficacy is needed to 1) Identify different training models and challenges that influence the OTS training, 2) Study the efficacy and control systems knowledge acquired using simulator training, its economic benefits and 3) To improve the competency of operators.

\section{ACKNOWLEDGEMENT}

The authors like to acknowledge different academic and industry professionals from Middle East, India, Norway, China and Europe for their inputs, discussions and support.

\section{REFERENCES}

[1] Glaser, D. C.; Garvey, M. B. (2014). Using simulator exercises for lifelong learning in the control room, $5^{\text {th }}$ Instructor Skills Conference, 12 pages

[2] Kallakuri, R.; Bahuguna, P. C.; Chawla, G. (2017). Riding the oil tides: strategies for oil and gas companies, Proceedings of the International Conference on Management of Infrastructure 2017, 198-211 
[3] Okoli, C.; Schabram, K. (2010). A Guide to conducting a systematic literature review of information systems research, Sprouts: Working Papers on Information Systems, Vol. 10, No. 26, 51 pages

[4] Nyumba, T. O.; Wilson, K.; Derrick, C. J.; Mukherjee, N. (2018). The use of focus group discussion methodology: insights from two decades of application in conservation, Methods in Ecology and Evolution, Vol. 9, No. 1, 20-32, doi:10.1111/2041-210X.12860

[5] Kluge, A.; Sauer, J.; Schüler, K.; Burkolter, D. (2009). Designing training for process control simulators: a review of empirical findings and current practices, Theoretical Issues in Ergonomics Science, Vol. 10, No. 6, 489-509, doi:10.1080/14639220902982192

[6] Komulainen, T. M.; Sannerud, A. R. (2018). Learning transfer through industrial simulator training: Petroleum industry case, Cogent Education, Vol. 5, No. 1, Paper 1554790, 19 pages, doi:10.1080/2331186X.2018.1554790

[7] Stetz, M. C.; Wildzunas, R. M.; Wiederhold, B. K.; Stetz. T. A.; Hunt, M. P. (2006). The usefulness of virtual reality stress inoculation training for military medical females: a pilot study, Annual Review of CyberTherapy and Telemedicine, Vol. 4, 51-58

[8] Baldwin, T. T.; Ford, J. K. (1988). Transfer of training: a review and directions for future research, Personnel Psychology, Vol. 41, No. 1, 63-105, doi:10.1111/j.1744-6570.1988.tb00632.x

[9] International Atomic Energy Agency (2004). Simulator instructor competence (Ch. 6), Chapman, C. R.; Yoder, J.; Mazour, T.; Kossilov, A. (Eds.), Use of Control Room Simulators for Training of Nuclear Power Plant Personnel, IAEA, Vienna, 23-26

[10] Burke, L. A.; Hutchins, H. M. (2007). Training transfer: an integrative literature review, Human Resource Development Review, Vol. 6, No. 3, 263-296, doi:10.1177/1534484307303035

[11] Tichon, J. G.; Wallis, G. M. (2010). Stress training and simulator complexity: why sometimes more is less, Behaviour \& Information Technology, Vol. 29, No. 5, 459-466, doi:10.1080/ 01449290903420184

[12] Vilela, F. F.; Leal, F.; Montevechi, J. A. B.; Piedade, D. D. C. (2020). Effect of human factor performance on the productivity of a manual assembly line, International Journal of Simulation Modelling, Vol. 19, No. 3, 365-374, doi:10.2507/IJSIMM19-3-508

[13] Stetz, M.; Wiederhold, B. K.; Wildzunas, R. (2006). Usefulness of virtual reality stress inoculation training for military medical females, CyberPsychology \& Behavior, Vol. 9, No. 6 (Abstracts from CyberTherapy 2006), 719-720

[14] Barnard, Y. F.; Veldhuis, G. J.; van Rooij, J. C. G. M. (2001), Evaluation in practice: identifying factors for improving transfer of training in technical domains, Studies in Educational Evaluation, Vol. 27, No. 3, 269-290, doi:10.1016/S0191-491X(01)00030-X

[15] Parasuraman, R.; Manzey, D. H. (2010). Complacency and bias in human use of automation: an attentional integration, Human Factors: The Journal of the Human Factors and Ergonomics Society, Vol. 52, No. 3, 381-410, doi:10.1177/0018720810376055

[16] Yamnill, S.; McLean, G. N. (2001). Theories supporting transfer of training, Human Resource Development Quarterly, Vol. 12, No. 2, 195-208, doi:10.1002/hrdq.7

[17] Patle, D. S.; Zainal, A.; Rangaiah, G. P. (2014). Operator training simulators in the chemical industry: review, issues, and future directions, Reviews in Chemical Engineering, Vol. 30, No. 2, 199-216, doi:10.1515/revce-2013-0027

[18] Moher, D.; Shamseer, L.; Clarke, M.; Ghersi, D.; Liberati, A.; Petticrew, M.; Shekelle, P.; Stewart, L. A. (2015). Preferred reporting items for systematic review and meta-analysis protocols (PRISMA-P) 2015 statement, Systematic Reviews, Vol. 4, Paper 1, 9 pages, doi:10.1186/20464053-4-1

[19] O'Neill, M. M.; Booth, S. R.; Lamb, J. T. (2018). Using NVivo ${ }^{\text {TM }}$ for literature reviews: the eight step pedagogy (N7+1), The Qualitative Report, Vol. 23, No. 13, Paper 3, 20 pages, doi: $10.46743 / 2160-3715 / 2018.3030$

[20] Joao, S.; Durand, A.; Schrevens, O. (2016). Plant operability optimization through dynamic simulation, a case study focused on phosphoric acid concentration unit, Procedia Engineering, Vol. 138, 378-389, doi:10.1016/j.proeng.2016.02.097

[21] Salas, E.; Wilson, K. A.; Priest, H. A.; Guthrie, J. W. (2006). Design, delivery, and evaluation of training systems, Salvendy, G. (Ed.), Handbook of Human Factors and Ergonomics, $3^{\text {rd }}$ edition, John Wiley \& Sons, Hoboken, 472-512, doi:10.1002/0470048204.ch18 
[22] Chirgwin, P. (2021). Skills development and training of future workers in mining automation control rooms, Computers in Human Behavior Reports, Vol. 4, Paper 100115, 10 pages, doi:10.1016/j.chbr.2021.100115

[23] Kallakuri, R.; Bahuguna, P. C.; Glaser, D. C.; Shivalkar, S. (2018). Study of effectiveness of operator training simulators in the oil and gas industry, Proceedings of the $59^{\text {th }}$ Conference on Simulation and Modelling, 79-86, doi:10.3384/ecp1815379

[24] Reising, D. V. C.; Bullemer, P. T. (2008). A direct perception, span-of-control overview display to support a process control operator's situation awareness: a practice-oriented design process, Proceedings of the Human Factors and Ergonomics Society Annual Meeting, Vol. 52, No. 4, $267-$ 271, doi: $10.1177 / 154193120805200415$

[25] Kumar, S.; Arekar, K.; Jain, R. (2016). The impact of effectiveness of the simulator training program on different factors of needs and interest of the training, $2^{\text {nd }}$ International Conference on Next Generation Computing Technologies (NGCT), 485-489, doi:10.1109/NGCT.2016.78774664

[26] ARC Advisory Group. Operations Management, from https://www.arcweb.com/technologies/ operations-management, accessed on 21-05-2021

[27] Philips, J. J. (2003). Isolating the effects of training, Philips, J. J. (Ed.), Return on Investment in Training and Performance Improvement Programs, $2^{\text {nd }}$ edition, Butterworth-Heinemann, Boston, 111-145, doi:10.1016/B978-0-7506-7601-4.50007-9

[28] Tverskoy, Y. S.; Golubev, A. V.; Nikonorov, A. N. (2011). The proof ground for automated process control systems of power stations: an efficient tool for training specialists and testing complex control systems, Thermal Engineering, Vol. 58, No. 10, 869-875, doi:10.1134/ $\underline{\mathrm{S} 0040601511100120}$

[29] Arango, I.; Herrera, A. (2020), Simulator with embedded intelligence focused on the design process, International Journal of Simulation Modelling, Vol. 19, No. 4, 619-630, doi:10.2507/IJSIMM19-4-533

[30] Marcano, L.; Yazidi, A.; Ferati, M.; Komulainen, T. (2017). Towards effective automatic feedback for simulator training, Proceedings of the $58^{\text {th }}$ Conference on Simulation and Modelling, 203-208, doi:10.3384/ecp17138203

[31] Meshkati, N. (2007). Lessons of the Chernobyl nuclear accident for sustainable energy generation: creation of the safety culture in nuclear power plants around the world, Energy Sources, Part A: Recovery, Utilization, and Environmental Effects, Vol. 29, No. 9, 807-815, doi:10.1080/ 00908310500280934

[32] Crabtree, B. F.; Miller, W. L. (1992). Doing Qualitative Research: Multiple Strategies, Sage Publications, Thousand Oaks

[33] IAEA TECDOC Series, No. 1392 (2018). Development of Instructors for Nuclear Power Plant Personnel Training, International Atomic Energy Agency, Vienna

[34] Roldan-Villasana, E. J. (2015). Importance of simulators, systematic approach to training, and integral instruction centres in the process industry, 2015 IEEE European Modelling Symposium, 157-162, doi:10.1109/EMS.2015.33

[35] Cropanzano, R.; Rupp, D. E.; Byrne, Z. S. (2003). The relationship of emotional exhaustion to work attitudes, job performance, and organizational citizenship behaviour, Journal of Applied Psychology, Vol. 88, No. 1, 160-169, doi:10.1037/0021-9010.88.1.160 